\title{
AN INEQUALITY CONNECTED WITH THE GROWTH OF ENTROPY
}

\author{
BY \\ W. A. DAY \\ Hertford College, Oxford, England
}

1. Introduction. If an isolated physical system has finite entropy $S(t)$, where $t$ is the time, then the second law of thermodynamics leads us to expect that

$$
\frac{d S}{d t} \geq 0,
$$

the inequality being strict unless the system is in equilibrium. We also expect that the system will approach equilibrium as $t$ increases and, hence, that

$$
\text { the limit } S(\infty)=\lim _{t \rightarrow \infty} S(t) \text { exists. }
$$

When we combine (1.1) with (1.2) we see that

$$
S(t) \leq S(\infty) .
$$

At this juncture it is natural to ask whether more can justifiably be said about the growth of entropy than the rather sparse information contained in the statements (1.1), (1.2), and (1.3).

With this question in mind, Ziff, Merajver, and Stell [1], conjectured, on the basis of numerical computation, that the entropy associated with the solution of a Boltzmann equation is completely monotone, that is to say

$$
(-1)^{n+1} \frac{d^{n} S}{d t^{n}} \geq 0 \quad(n=1,2,3, \ldots),
$$

but subsequently Lieb [2] showed, by means of an analytical argument, that the conjecture is false.

Again, Simons [3] has claimed that, according to the classical theory of heat conduction, the entropy of a thermally insulated rigid conductor must be completely monotone. Simons' argument is analytical rather than numerical in character but it depends upon making an approximation to the rate of generation of entropy; granted the approximation, Simons' conclusion is valid but, as I have shown in $[4, \mathrm{Ch} .5]$, the conclusion is not valid if the correct formula for the rate of generation of entropy is used.

Received November 4, 1992.

1991 Mathematics Subject Classification. Primary 35K, 80.

(C)1995 Brown University 
The purpose of the present paper is to demonstrate that a stronger inequality than (1.1) can be proved for the parabolic equation which describes heat conduction in an inhomogeneous rod whose ends are insulated. To be more specific, it will be shown that the relations (1.2) and (1.3) hold while (1.1) can be replaced by the inequality

$$
\frac{d S}{d t}(t) \geq \lambda(S(\infty)-S(t))
$$

where $\lambda$ is a known positive constant.

It is an immediate consequence of (1.4) that the approach of the entropy to its limiting value is, in fact, exponential:

$$
S(t)=S(\infty)+O(\exp (-\lambda t)) \quad \text { as } t \rightarrow \infty .
$$

In order to prove (1.4) it is necessary to start by proving an analytic inequality which appears to be new and which is of interest in its own right.

2. An analytic inequality. The required inequality is set out in the following lemma.

Lemma. On the interval $[0, L]$ let $p(x)$ be positive and continuous, and let $f(x)$ be positive and have a continuous derivative $f^{\prime}(x)$. Then

$$
\log \left[\frac{\int_{0}^{L} p f d x}{\int_{0}^{L} p d x}\right] \leq \frac{\int_{0}^{L} p \log f d x}{\int_{0}^{L} p d x}+\frac{1}{2 \pi^{2}} \int_{0}^{L} p d x \int_{0}^{L} \frac{1}{p}\left(\frac{f^{\prime}}{f}\right)^{2} d x,
$$

the constant $1 /\left(2 \pi^{2}\right)$ being the best possible.

The inequality can be regarded as providing a converse to the familiar inequality

$$
\exp \left[\frac{\int_{0}^{L} p \log f d x}{\int_{0}^{L} p d x}\right] \leq \frac{\int_{0}^{L} p f d x}{\int_{0}^{L} p d x}
$$

between the weighted geometric and arithmetic means of $f$ [5, Theorem 184].

On making the substitution $f(x)=\exp (\varepsilon g(x))$ in (2.1), and considering the asymptotic behaviour of each side as $\varepsilon \rightarrow 0$, we arrive at an inequality of the Wirtinger type, namely

$$
\frac{\int_{0}^{L} p g^{2} d x}{\int_{0}^{L} p d x} \leq\left(\frac{\int_{0}^{L} p g d x}{\int_{0}^{L} p d x}\right)^{2}+\frac{1}{\pi^{2}} \int_{0}^{L} p d x \int_{0}^{L} \frac{1}{p}\left(g^{\prime}\right)^{2} d x .
$$

The proof of the lemma involves the construction of certain solutions of the heat equation.

It will be assumed for the moment that

$$
f(x) \text { has a continuous second derivative and } f^{\prime}(0)=f^{\prime}(L)=0,
$$

and that

$$
\text { the weight function } p(x) \equiv 1 \text { for } 0 \leq x \leq L .
$$

These additional restrictions will be removed at a later stage. 
First, let the domain of definition of $f(x)$ be extended to the interval $[-L, L]$ by requiring $f(x)$ to be an even function, that is to say $f(-x)=f(x)$. The extended function has a Fourier development

$$
f(x) \sim c_{0}+\sum_{n=1}^{\infty} c_{n} \cos \left(\frac{n \pi x}{L}\right) .
$$

Moreover, the extended function is continuously differentiable on $[-L, L]$, by virtue of the restriction $f^{\prime}(0)=0$, and it satisfies the condition $f(-L)=f(L)$. Thus, when $n \rightarrow \infty$, the partial sums

$$
f_{n}(x)=c_{0}+\sum_{k=1}^{n} c_{k} \cos \left(\frac{k \pi x}{L}\right)
$$

converge to $f(x)$, the convergence being uniform on $[-L, L]$. Since $f(x)$ is bounded from below by a positive constant there must exist positive constants $A$ and $B$, and a positive integer $n_{0}$, such that

$$
0<A \leq f_{n}(x) \leq B \text { for every } x \text { in }[0, L] \text { and every } n>n_{0} \text {. }
$$

The derivative of $f(x)$ has the Fourier development

$$
f^{\prime}(x) \sim-\sum_{n=1}^{\infty} \frac{n \pi c_{n}}{L} \sin \left(\frac{n \pi x}{L}\right) .
$$

Furthermore, $f^{\prime}(x)$ is continuous and piecewise continuously differentiable on $[-L, L]$, and it satisfies the conditions $f^{\prime}(-L)=0=f^{\prime}(L)$. Hence $f_{n}^{\prime}(x) \rightarrow f^{\prime}(x)$ uniformly on $[-L, L]$ when $n \rightarrow \infty$, and, in particular, there must be a positive constant $C$, and a positive integer which can be taken to be the $n_{0}$ of (2.5), such that

$$
\left|f_{n}^{\prime}(x)\right| \leq C \text { for every } x \text { in }[0, L] \text { and every } n>n_{0} .
$$

For any $n>n_{0}$, we introduce the finite sum

$$
u_{n}(x, t)=c_{0}+\sum_{k=1}^{n} c_{k} \cos \left(\frac{k \pi x}{L}\right) \exp \left(-\frac{k^{2} \pi^{2} t}{L^{2}}\right),
$$

which has derivatives of all orders on the strip $[0, L] \times(-\infty, \infty)$ and satisfies the heat equation

$$
\frac{\partial^{2} u_{n}}{\partial x^{2}}=\frac{\partial u_{n}}{\partial t}
$$

the boundary conditions

$$
\frac{\partial u_{n}}{\partial x}(0, t)=\frac{\partial u_{n}}{\partial x}(L, t)=0 \text { for }-\infty<t<\infty,
$$

and the initial condition

$$
u_{n}(x, 0)=f_{n}(x) \quad \text { for } 0 \leq x \leq L .
$$


By virtue of the identity

$$
\begin{aligned}
& \int_{-\infty}^{\infty} \exp \left(-\frac{y^{2}}{4 t}\right) \cos \left(\frac{k \pi}{L}(x-y)\right) d y \\
&=\sqrt{4 \pi t} \exp \left(-\frac{k^{2} \pi^{2} t}{L^{2}}\right) \cos \left(\frac{k \pi x}{L}\right) \quad(t>0),
\end{aligned}
$$

$u_{n}(x, t)$ can be expressed as the integral

$$
\frac{1}{\sqrt{4 \pi t}} \int_{-\infty}^{\infty} \exp \left(-\frac{y^{2}}{4 t}\right) f_{n}(x-y) d y \quad(t>0)
$$

and it is clear from this that $u_{n}(x, t)>0$ on the half-strip $[0, L] \times(0, \infty)$. Since the initial condition (2.10) holds, with $f_{n}(x)>0$, it must be that $u_{n}(x, t)>0$ on $[0, L] \times[0, \infty)$. A continuity argument now implies that there exists $\tau<0$ such that $u_{n}(x, t)>0$ on the larger half-strip $[0, L] \times(\tau, \infty)$ and, hence, that the entropy

$$
S_{n}(t)=\int_{0}^{L} \log u_{n} d x
$$

is defined for every $t>\tau$.

We note that

$$
S_{n}(0)=\int_{0}^{L} \log f_{n} d x .
$$

We also note that, when $t \rightarrow \infty$,

$$
u_{n}(x, t) \rightarrow c_{0}=\frac{1}{2 L} \int_{-L}^{L} f d x=\frac{1}{L} f d x
$$

where $\left|u_{n}(x, t)\right| \leq\left|c_{0}\right|+\sum_{k=1}^{n}\left|c_{k}\right|$ on $[0, L] \times(-\infty, \infty)$. Thus the dominated convergence theorem ensures that

$$
S_{n}(t) \rightarrow L \log c_{0}=L \log \left(\frac{1}{L} \int_{0}^{L} f d x\right) \quad \text { as } t \rightarrow \infty .
$$

In a similar way we can see that

$$
\frac{d S_{n}}{d t}(t) \rightarrow 0 \text { as } t \rightarrow \infty .
$$

At this stage it is convenient to introduce the function $v_{n}(x, t)=\log u_{n}(x, t)$, which is defined on $[0, L] \times(\tau, \infty)$, has derivatives of all orders, and satisfies the differential equation

$$
\frac{\partial^{2} v_{n}}{\partial x^{2}}+\left(\frac{\partial v_{n}}{\partial x}\right)^{2}=\frac{\partial v_{n}}{\partial t}
$$

the boundary conditions

$$
\frac{\partial v_{n}}{\partial x}(0, t)=\frac{\partial v_{n}}{\partial x}(L, t)=0 \text { for } t>\tau,
$$

and the initial condition

$$
v_{n}(x, 0)=\log f_{n}(x) \text { for } 0 \leq x \leq L .
$$


Since the boundary conditions (2.15) are in force, a known inequality [5, Theorem 257] assures us that

$$
\frac{\pi^{2}}{L^{2}} \int_{0}^{L}\left(\frac{\partial v_{n}}{\partial x}\right)^{2} d x \leq \int_{0}^{L}\left(\frac{\partial^{2} v_{n}}{\partial x^{2}}\right)^{2} d x
$$

The entropy can be written in terms of $v_{n}(x, t)$ as the integral

$$
S_{n}(t)=\int_{0}^{L} v_{n} d x
$$

and if we calculate the derivative of $S_{n}(t)$ and appeal to the differential equation (2.14) and the boundary conditions $(2.15)$ we find that

$$
\frac{d S_{n}}{d t}=\int_{0}^{L} \frac{\partial v_{n}}{\partial t} d x=\int_{0}^{L}\left[\frac{\partial^{2} v_{n}}{\partial x^{2}}+\left(\frac{\partial v_{n}}{\partial x}\right)^{2}\right] d x=\int_{0}^{L}\left(\frac{\partial v_{n}}{\partial x}\right)^{2} d x
$$

In particular,

$$
\frac{d S_{n}}{d t}(0)=\int_{0}^{L}\left(\frac{f_{n}^{\prime}}{f_{n}}\right)^{2} d x
$$

On calculating a further derivative, and appealing to the boundary conditions (2.15), we see that

$$
\begin{aligned}
\frac{d^{2} S_{n}}{d t^{2}} & =2 \int_{0}^{L} \frac{\partial v_{n}}{\partial x} \frac{\partial^{2} v_{n}}{\partial x \partial t} d x \\
& =2 \int_{0}^{L}\left[\frac{\partial}{\partial x}\left(\frac{\partial v_{n}}{\partial x} \frac{\partial v_{n}}{\partial t}\right)-\frac{\partial^{2} v_{n}}{\partial x^{2}} \frac{\partial v_{n}}{\partial t}\right] d x \\
& =-2 \int_{0}^{L} \frac{\partial^{2} v_{n}}{\partial x^{2}} \frac{\partial v_{n}}{\partial t} d x .
\end{aligned}
$$

Thus if we substitute for $\partial v_{n} / \partial t$ from Eq. (2.14) and invoke the boundary conditions yet again we have

$$
\begin{aligned}
\frac{d^{2} S_{n}}{d t^{2}} & =-2 \int_{0}^{L}\left[\left(\frac{\partial^{2} v_{n}}{\partial x^{2}}\right)^{2}+\frac{\partial^{2} v_{n}}{\partial x^{2}}\left(\frac{\partial v_{n}}{\partial x}\right)^{2}\right] d x \\
& =-2 \int_{0}^{L}\left[\left(\frac{\partial^{2} v_{n}}{\partial x^{2}}\right)^{2}+\frac{1}{3} \frac{\partial}{\partial x}\left\{\left(\frac{\partial v_{n}}{\partial x}\right)^{3}\right\}\right] d x
\end{aligned}
$$

and therefore,

$$
\frac{d^{2} S_{n}}{d t^{2}}=-2 \int_{0}^{L}\left(\frac{\partial^{2} v_{n}}{\partial x^{2}}\right)^{2} d x
$$

If we combine Eqs. (2.18) and (2.20) with the inequality (2.17) we arrive at the inequality

$$
\frac{d S_{n}}{d t}+\frac{L^{2}}{2 \pi^{2}} \frac{d^{2} S_{n}}{d t^{2}} \leq 0 \text { for } t>\tau
$$


Integration with respect to $t$ now yields the inequality

$$
S_{n}(t)+\frac{L^{2}}{2 \pi^{2}} \frac{d S_{n}}{d t}(t) \leq S_{n}(0)+\frac{L^{2}}{2 \pi^{2}} \frac{d S_{n}}{d t}(0) \text { for } t>0,
$$

and, on letting $t \rightarrow \infty$ and appealing to the relations (2.11), (2.12), (2.13), and (2.19), we conclude that

$$
L \log \left(\frac{1}{L} \int_{0}^{L} f d x\right) \leq \int_{0}^{L} \log f_{n} d x+\frac{L^{2}}{2 \pi^{2}} \int_{0}^{L}\left(\frac{f_{n}^{\prime}}{f_{n}}\right)^{2} d x .
$$

Since $f_{n}(x) \rightarrow f(x)$ and $f_{n}^{\prime}(x) \rightarrow f^{\prime}(x)$ as $n \rightarrow \infty$, and since the conditions (2.5) and (2.6) hold, the dominated convergence theorem assures us that, as $n \rightarrow \infty$,

$$
\int_{0}^{L} \log f_{n} d x \rightarrow \int_{0}^{L} \log f d x, \quad \int_{0}^{L}\left(\frac{f_{n}^{\prime}}{f_{n}}\right)^{2} d x \rightarrow \int_{0}^{L}\left(\frac{f^{\prime}}{f}\right)^{2} d x .
$$

Thus, on letting $n \rightarrow \infty$ and dividing through by $L$, we conclude that the inequality

$$
\log \left(\frac{1}{L} \int_{0}^{L} f d x\right) \leq \int_{0}^{L} \log f d x+\frac{L}{2 \pi^{2}} \int_{0}^{L}\left(\frac{f^{\prime}}{f}\right)^{2} d x
$$

holds provided that $f(x)$ is positive and satisfies the conditions (2.2).

Next we shall show that the additional restrictions (2.2) are, in fact, irrelevant and that (2.22) is satisfied provided only that $f(x)$ is positive and has a continuous derivative. Let $\varepsilon>0$ be arbitrary and choose a function $g(x)$ that has a continuous derivative, that satisfies the conditions $g(0)=g(L)=0$, and that approximates $f^{\prime}(x)$ in the sense that

$$
\left\|f^{\prime}-g\right\|<\varepsilon,
$$

the norm being the $L^{2}$-norm, that is to say,

$$
\left\|f^{\prime}-g\right\|=\sqrt{\int_{0}^{L}\left(f^{\prime}-g\right)^{2} d x}
$$

If we introduce the function

$$
h(x)=f(0)+\int_{0}^{x} g(s) d s
$$

then $h^{\prime}(x)=g(x)$, and $h(x)$ is twice continuously differentiable and satisfies the conditions $h^{\prime}(0)=h^{\prime}(L)=0$.

Since $f(x)$ has a continuous derivative, the fundamental theorem of the calculus implies that

$$
f(x)=f(0)+\int_{0}^{x} f^{\prime}(s) d s,
$$


and this and the Cauchy-Schwarz inequality tell us that

$$
\begin{aligned}
|f(x)-h(x)| & =\left|\int_{0}^{x}\left(f^{\prime}(s)-g(s)\right) d s\right| \\
& \leq \sqrt{x} \sqrt{\int_{0}^{x}\left(f^{\prime}(s)-g(s)\right)^{2} d s} \\
& \leq \sqrt{L} \cdot \varepsilon
\end{aligned}
$$

for every $x$ in $[0, L]$. Thus $h(x)>0$ on $[0, L]$ provided that $\varepsilon<m / \sqrt{L}$, where $m(>0)$ is the minimum value attained by $f(x)$ on $[0, L]$. Thus, from what has been proved already, $h(x)$ must satisfy the inequality

$$
\log \left(\frac{1}{L} \int_{0}^{L} h d x\right) \leq \frac{1}{L} \int_{0}^{L} \log h d x+\frac{L}{2 \pi^{2}} \int_{0}^{L}\left(\frac{h^{\prime}}{h}\right)^{2} d x .
$$

Each of the terms in (2.25) can be estimated with the aid of the inequalities (2.23) and (2.24). According to (2.24)

$$
\frac{1}{L} \int_{0}^{L} h d x \geq \frac{1}{L} \int_{0}^{L} f d x-\sqrt{L} \cdot \varepsilon
$$

while the elementary inequality $\log (1+x) \leq x \quad(x>-1)$ tells us that

$$
\begin{aligned}
\log h & =\log f+\log \left(1+\frac{h-f}{f}\right) \\
& \leq \log f+\frac{h-f}{f} \\
& \leq \log f+\frac{\sqrt{L} \cdot \varepsilon}{f} \\
& \leq \log f+\frac{\sqrt{L} \cdot \varepsilon}{m}
\end{aligned}
$$

and, hence, that

$$
\frac{1}{L} \int_{0}^{L} \log h d x \leq \frac{1}{L} \int_{0}^{L} \log f d x+\frac{\sqrt{L} \cdot \varepsilon}{m} .
$$

If we choose $\varepsilon<m /(2 \sqrt{L})$ then $h(x)>m / 2$ on $\left[0,{ }^{r}\right]$ and, therefore,

$$
\begin{aligned}
\left|\frac{f^{\prime}}{f}-\frac{h^{\prime}}{h}\right| & =\left|\frac{f^{\prime}-h^{\prime}}{h}-\frac{(f-h) f^{\prime}}{f h}\right| \\
& \leq \frac{\left|f^{\prime}-h^{\prime}\right|}{h}+\frac{\left|(f-h) f^{\prime}\right|}{f h} \\
& \leq \frac{2}{m}\left|f^{\prime}-h^{\prime}\right|+\frac{2}{m^{2}} \sqrt{L} \cdot \varepsilon\left|f^{\prime}\right| \\
& =\frac{2}{m}\left|f^{\prime}-g\right|+\frac{2}{m^{2}} \sqrt{L} \cdot \varepsilon\left|f^{\prime}\right| .
\end{aligned}
$$

Hence

$$
\left\|\frac{f^{\prime}}{f}-\frac{h^{\prime}}{h}\right\| \leq \frac{2}{m}\left\|f^{\prime}-g\right\|+\frac{2}{m^{2}} \sqrt{L} \cdot \varepsilon\left\|f^{\prime}\right\| \leq \frac{2 \varepsilon}{m}+\frac{2 \sqrt{L} \cdot \varepsilon}{m^{2}}\left\|f^{\prime}\right\|
$$


and

$$
\left\|\frac{h^{\prime}}{h}\right\| \leq\left\|\frac{f^{\prime}}{f}\right\|+\frac{2 \varepsilon}{m}+\frac{2 \sqrt{L} \cdot \varepsilon}{m^{2}}\left\|f^{\prime}\right\| .
$$

If we combine (2.25) with (2.26), (2.27), and (2.28), we see that

$$
\begin{aligned}
\log \left(\frac{1}{L} \int_{0}^{L} f d x-\sqrt{L} \cdot \varepsilon\right) \leq & \frac{1}{L} \int_{0}^{L} \log f d x+\frac{\sqrt{L} \cdot \varepsilon}{m} \\
& +\frac{L}{2 \pi^{2}}\left[\left\|\frac{f^{\prime}}{f}\right\|+\frac{2 \varepsilon}{m}+\frac{2 \sqrt{L} \cdot \varepsilon}{m^{2}}\left\|f^{\prime}\right\|\right]^{2}
\end{aligned}
$$

for every $\varepsilon$ in $0<\varepsilon<m /(2 \sqrt{L})$, and, on letting $\varepsilon \rightarrow 0$, we see that the inequality (2.22) must hold whenever $f(x)$ is positive and has a continuous derivative.

Next we remove the restriction (2.3) on the weight function $p(x)$. To do so let $p(x)$ be any function that is positive and continuous on $[0, L]$, and let

$$
\xi(x)=\int_{0}^{x} p(s) d s, \quad M=\xi(L) .
$$

Since $\xi(x)$ is strictly increasing and continuously differentiable on $[0, L]$, the relation $y=\xi(x)$ can be inverted to yield a relation $x=\eta(y)$, where $\eta(y)$ is strictly increasing and continuously differentiable on $[0, M]$. If we set $g(y)=f(\eta(y))$ then $g(y)$ is positive and continuously differentiable on the interval $[0, M]$, and, by virtue of what we have already proved,

$$
\log \left[\frac{1}{M} \int_{0}^{M} g d y\right] \leq \frac{1}{M} \int_{0}^{M} \log g d y+\frac{M}{2 \pi^{2}} \int_{0}^{M}\left(\frac{g^{\prime}}{g}\right)^{2} d y
$$

However,

$$
M=\int_{0}^{L} p(x) d x
$$

and if we make the change of variable $y=\xi(x)$ in the integrals that occur in (2.30), and remember that

$$
\xi^{\prime}(x)=p(x), \quad \eta^{\prime}(y)=\frac{1}{p(\eta(y))}, \quad g^{\prime}(y)=\frac{f^{\prime}(\eta(y))}{p(\eta(y))},
$$

we see that

$$
\begin{gathered}
\int_{0}^{M} g(y) d y=\int_{0}^{L} p(x) f(x) d s, \\
\int_{0}^{M} \log g(y) d y=\int_{0}^{L} p(x) \log f(x) d x, \\
\int_{0}^{M}\left(\frac{g^{\prime}(y)}{g(y)}\right)^{2} d y=\int_{0}^{L} \frac{1}{p(x)}\left(\frac{f^{\prime}(x)}{f(x)}\right)^{2} d x .
\end{gathered}
$$

Thus, on substituting from (2.31), (2.32), (2.33), and (2.34), into (2.30), we arrive at the required inequality $(2.1)$ whose validity is now seen not to depend upon the additional restrictions (2.2) and (2.3). 
It remains to be shown that the constant $1 /\left(2 \pi^{2}\right)$ is best possible. Suppose, to the contrary, that an inequality

$$
\log \left[\frac{\int_{0}^{L} p f d x}{\int_{0}^{L} p d x}\right] \leq \frac{\int_{0}^{L} p \log f d x}{\int_{0}^{L} p d x}+\kappa \int_{0}^{L} p d x \int_{0}^{L} \frac{1}{p}\left(\frac{f^{\prime}}{f}\right)^{2} d x
$$

holds for all positive and continuously differentiable functions $f(x)$, and with a constant $\kappa<1 /\left(2 \pi^{2}\right)$. If we make the choice

$$
f(x)=\exp \{\varepsilon \cos (\pi \xi(x) / \xi(L))\},
$$

where $\xi(x)$ is defined by (2.29) and $\varepsilon$ is any positive number, then

$$
\begin{aligned}
\int_{0}^{L} p \log f d x & =\varepsilon \int_{0}^{L} \xi^{\prime}(x) \cos (\pi \xi(x) / \xi(L)) d x \\
& =\frac{\varepsilon \xi(L)}{\pi}[\sin (\pi \xi(x) / \xi(L))]_{x=0}^{L}=0
\end{aligned}
$$

and

$$
\begin{aligned}
\int_{0}^{L} \frac{1}{p}\left(\frac{f^{\prime}}{f}\right)^{2} d x & =\left(\frac{\varepsilon \pi}{\xi(L)}\right)^{2} \int_{0}^{L} \xi^{\prime}(x) \sin ^{2}(\pi \xi(x) / \xi(L)) d x \\
& =\left(\frac{\varepsilon \pi}{\xi(L)}\right)^{2} \cdot \frac{1}{2} \int_{0}^{L} \xi^{\prime}(x)\left\{1-\cos \left(\frac{2 \pi \xi(x)}{\xi(L)}\right)\right\} d x \\
& =\frac{(\varepsilon \pi)^{2}}{2 \xi(L)}=\frac{(\varepsilon \pi)^{2}}{2 \int_{0}^{L} p d x} .
\end{aligned}
$$

Moreover, if we expand $f(x)$ in powers of $\varepsilon$, and integrate term-by-term, we can readily deduce that when $\varepsilon \rightarrow 0$

$$
\begin{aligned}
\frac{\int_{0}^{L} p f d x}{\int_{0}^{L} p d x} & =\frac{1}{\int_{0}^{L} p d x} \int_{0}^{L} \xi^{\prime}(x)\left[1+\varepsilon \cos \left(\frac{\pi \xi(x)}{\xi(L)}\right)+\frac{1}{2} \varepsilon^{2} \cos ^{2}\left(\frac{\pi \xi(x)}{\xi(L)}\right)\right] d x+o\left(\varepsilon^{2}\right) \\
& =1+\frac{1}{4} \varepsilon^{2}+o\left(\varepsilon^{2}\right)
\end{aligned}
$$

and, therefore, that

$$
\log \left[\frac{\int_{0}^{L} p f d x}{\int_{0}^{L} p d x}\right]=\frac{1}{4} \varepsilon^{2}+o\left(\varepsilon^{2}\right) .
$$

Thus, if we substitute from (2.36), (2.37), and (2.38), into (2.35), divide through by $\varepsilon^{2}$, and let $\varepsilon \rightarrow 0$, we deduce that

$$
\frac{1}{4} \leq \frac{\kappa \pi^{2}}{2}
$$

that is to say $\kappa \geq 1 /\left(2 \pi^{2}\right)$, contrary to hypothesis. Hence the constant $1 /\left(2 \pi^{2}\right)$ is best possible and the proof of the lemma is complete. 
3. An application to the growth of entropy. The analytic inequality of the Lemma has an application to the growth of entropy associated with a function $u(x, t)$ that is defined on the half-strip $[0, L] \times[0, \infty]$, and that is a solution of the parabolic equation

$$
\frac{\partial}{\partial x}\left(a(x) \frac{\partial u}{\partial x}\right)=b(x) \frac{\partial u}{\partial t}, \quad 0<x<L \text { and } t>0
$$

and satisfies the boundary conditions

$$
\frac{\partial u}{\partial x}(0, t)=\frac{\partial u}{\partial x}(L, t)=0, \quad t>0
$$

and an initial condition

$$
u(x, 0)=\varphi(x), \quad 0 \leq x \leq L .
$$

It is assumed, in addition, that

$$
u(x, t)>0 \text { on }[0, L] \times[0, \infty),
$$

and if this is so the entropy is defined by the relation

$$
S(t)=\int_{0}^{L} b \log u d x .
$$

Here $u(x, t)$ can be thought of as the temperature, measured on the Kelvin scale, of an inhomogeneous rod whose ends are insulated. The variable coefficients $a(x)$ and $b(x)$ are, respectively, the thermal conductivity and the heat capacity of unit length; each is required to be positive and continuous on $[0, L]$.

The solution to the initial-and-boundary value problem can be constructed using an expansion in eigenfunctions. Thus, let $\mu_{0}, \mu_{1}, \mu_{2}, \ldots$, ordered so that $\mu_{0}<$ $\mu_{1}<\mu_{2}<\cdots$, be the eigenvalues associated with the Sturm-Liouville problem

$$
-\left(a(x) \psi^{\prime}(x)\right)^{\prime}=\mu b(x) \psi(x), \quad \psi^{\prime}(0)=\psi^{\prime}(L)=0,
$$

and let $\psi_{0}(x), \psi_{1}(x), \psi_{2}(x), \ldots$ be the corresponding eigenfunctions. In fact, the least eigenvalue $\mu_{0}=0$ and, without loss of generality, we may take it that $\psi_{0} \equiv 1$ on $[0, L]$. The eigenfunctions satisfy familiar orthogonality relations, namely

$$
\int_{0}^{L} b \psi_{m} \psi_{n} d x \equiv 0 \text { if } m \neq n \text {. }
$$

Now let the series

$$
\sum_{n=0}^{\infty} c_{n} \psi_{n}(x)
$$

be the generalized Fourier development of the initial temperature $\varphi(x)$. The leading coefficient, which is of especial significance in the present context, is

$$
c_{0}=\frac{\int_{0}^{L} b \varphi d x}{\int_{0}^{L} b d x},
$$

and the solution of the initial-and-boundary value problem is

$$
u(x, t)=\sum_{n=0}^{\infty} c_{n} \psi_{n}(x) \exp \left(-\mu_{n} t\right) \text {. }
$$


As $t \rightarrow \infty, u(x, t) \rightarrow c_{0}$ and the entropy tends to the limiting value

$$
S(\infty)=\int_{0}^{L} b \log c_{0} d x=\int_{0}^{L} b d x \log \left[\int_{0}^{L} b \varphi d x / \int_{0}^{L} b d x\right] .
$$

Since the differential equation (3.1) and the boundary conditions (3.2) imply that

$$
\frac{d}{d t} \int_{0}^{L} b u d x=0
$$

and, hence, that

$$
\int_{0}^{L} b u d x=\int_{0}^{L} b \varphi d x \text { for } t \geq 0
$$

the initial temperature $\varphi(x)$ can be replaced by $u(x, t)$ in (3.6) to get

$$
S(\infty)=\int_{0}^{L} b d x \log \left[\int_{0}^{L} b u d x / \int_{0}^{L} b d x\right]
$$

In the present circumstances we can readily confirm the consequences of the second law of thermodynamics to which we alluded in the introduction. On dividing both sides of the differential equation $(3.1)$ by $u(x, t)$, which, it will be recalled, has been assumed to be positive, we deduce that

$$
\frac{\partial}{\partial x}\left(\frac{a}{u} \frac{\partial u}{\partial x}\right)+\frac{a}{u^{2}}\left(\frac{\partial u}{\partial x}\right)^{2}=\frac{b}{u} \frac{\partial u}{\partial t}
$$

and, therefore, that

$$
\frac{d S}{d t}=\int_{0}^{L} \frac{b}{u} \frac{\partial u}{\partial t} d x=\int_{0}^{L} \frac{a}{u^{2}}\left(\frac{\partial u}{\partial x}\right) \geq 0
$$

in agreement with the inequality (1.1). We have seen already that the condition (1.2) is satisfied and it then follows immediately that the inequality (1.3) holds for every $t \geq 0$. In view of (3.5) and (3.7), the inequality (1.3) is equivalent to the well-known inequality between the weighted geometric and arithmetic means of $u(x, t)$, with weight function $b(x)$.

We turn to proving the following theorem.

THEOREM. The inequality (1.1) can be replaced by the stronger inequality

$$
\frac{d S}{d t}(t) \geq \lambda(S(\infty)-S(t)), \quad t \geq 0
$$

where $\lambda$ is the positive constant

$$
\frac{2 \pi^{2} \min \{a(x) b(x): 0 \leq x \leq L\}}{\left(\int_{0}^{L} b d x\right)^{2}} .
$$

It might be thought possible to prove the theorem by calculating the second derivative of the entropy and attempting to derive a counterpart to the inequality (2.21), which, as we have seen, is valid for the heat equation. However, such an approach appears to break down when the coefficients $a(x)$ and $b(x)$ vary with $x$, and we are forced instead to argue directly from the lemma. 
Indeed, if we replace $f(x)$ by $u(x, t)$, with any $t \geq 0$, and replace the weight function $p(x)$ by $b(x)$, we see that

$$
\log \left[\frac{\int_{0}^{L} b u d x}{\int_{0}^{L} b d x}\right] \leq \frac{\int_{0}^{L} b \log u d x}{\int_{0}^{L} b d x}+\frac{1}{2 \pi^{2}} \int_{0}^{L} b d x \int_{0}^{L} \frac{1}{b u^{2}}\left(\frac{\partial u}{\partial x}\right)^{2} d x .
$$

Thus if we multiply both sides by $\int_{0}^{L} b d x$ and invoke (3.5) and (3.7) we have

$$
S(\infty) \leq S(t)+\frac{1}{2 \pi^{2}}\left(\int_{0}^{L} b d x\right)^{2} \int_{0}^{L} \frac{1}{b u^{2}}\left(\frac{\partial u}{\partial x}\right)^{2} d x .
$$

On the other hand, Eq. (3.8) tells us that

$$
\frac{d S}{d t}=\int_{0}^{L} \frac{a b}{b u^{2}}\left(\frac{\partial u}{\partial x}\right)^{2} d x \geq \min (a b) \int_{0}^{L} \frac{1}{b u^{2}}\left(\frac{\partial u}{\partial x}\right)^{2} d x
$$

and when we combine (3.11) and (3.2) we arrive at the desired inequality (3.9), with $\lambda$ defined as in (3.10).

\section{REFERENCES}

[1] R. M. Ziff, S. D. Merajver, and G. Stell, Approach to equilibrium of a Boltzmann-equation solution, Phys. Rev. Lett. 47, 1493-1496 (1981)

[2] E. H. Lieb, Comment upon “Approach to equilibrium of a Boltzmann-equation solution", Phys. Rev. Lett. 48, 1057 (1982)

[3] S. Simons, On entropy production for an isolated system, J. Phys. A. (General Physics) 4, 11-16 (1971)

[4] W. A. Day, Entropy and partial differential equations, Pitman Research Notes in Mathematics Series No. 295, Longman Scientific and Technical, Harlow, UK, 1993

[5] G. H. Hardy, J. E. Littlewood, and'G. Pólya, Inequalities, 2nd edition, Cambridge University Press, London and New York, 1952 